Tohoku J. Exp. Med., 2004, 204, 37-44

\title{
Progression and Direction of Contractures of Knee Joints Following Spinal Cord Injury in the Rat
}

\author{
Hideki Moriyama, Osamu Yoshimura, ${ }^{1}$ Hitoshi Sunahori, Haruko Nitta, \\ Hidetaka Imakita, Yukari SaKa, Hiroshi Maejima ${ }^{1}$ and Yoshiko Tobimatsu ${ }^{2}$ \\ Graduate School of Health Science, ${ }^{1}$ Institute of Health Sciences, School of \\ Medicine, Hiroshima University, Hiroshima 734-8551, and ${ }^{2}$ Department of \\ Rehabilitation Medicine, Graduate School, Tohoku University, Sendai 980-8575
}

Moriyama, H., Yoshimura, O., Sunahori, S., Nitta, H., Imakita, H., Saka, Y., Maejima, H. and Tobimatsu, Y. Progression and Direction of Contractures Following Spinal Cord Injury in Rat Knee Joints. Tohoku J. Exp. Med., 2004, 204 (1), 37-44 — Joint contractures following central nervous system injuries remain a prevalent and significant complication, but no reports are available on evidence of contracture formation over time. The objective of this study was to determine the rate of contracture progression and the direction of loss in joint movement following spinal cord injuries (SCI). Forty-eight female Wistar rats were used. Twenty-four experimental rats underwent a spinal cord transection at the level of T8 and 24 control rats underwent a sham-operation. The animals were studied at each of 5 time points: 2, 4, 8, 12, 16, and 24 weeks after surgical intervention. The degree of contractures was assessed by measuring the femorotibial angle on both hindlimbs with the use of a goniometer. Knee joint motion was measured for flexion and extension direction. Knee flexion contractures developed in all experimental rats. The restriction in motion progressed during the first 12 weeks and plateaued thereafter. The contractures were produced almost exclusively by a loss in the extension range of motion. This study defined the time course that contracture progression was more rapid in the early stage after SCI and stabilized in the later stage of injury. Contractures following SCI occurred in flexion at the knees and resulted from a loss of extension. These findings should help guide timely treatment and provide a better understanding of contracture development. — contracture; range of motion, articular; spinal cord injuries; rats

(C) 2004 Tohoku University Medical Press

Received May 7, 2004; revision accepted for publication June 28, 2004.

Address for reprints: Hideki Moriyama, Graduate School of Health Science, Hiroshima University, 1-2-3 Kasumi, Minami-ku, Hiroshima 734-8551, Japan.

e-mail: hidekimr@hiroshima-u.ac.jp

Present address for O. Yoshimura, Hiroshima City Counseling Center for the Physically Disabled, 2-1-5 Hikari-machi, Higashi-ku, Hiroshima 732-0052, Japan, for H. Sunahori, Hiroshima Prefectural Rehabilitation Center, 295-3 Saijou-cho, Higashi-Hiroshima 732-0052, Japan, and for Y. Tobimatsu, Graduate School of Health Science, Hiroshima University, 1-2-3 Kasumi, Minami-ku, Hiroshima 734-8551, Japan. 
Joint contractures are defined as limitations in the normal range of motion (ROM) of a joint. Recent advances in clinical management have led to a better understanding of their nature, and several treatment modalities are in use to reduce their severity (Farmer and James 2001). However, contractures following central nervous system (CNS) injuries remain a prevalent and significant complication.

A majority of information obtained on contracture formation is based on animal models with contractures produced experimentally. These studies have documented joint changes over time after immobilization by internal fixation (with plate or splint), external fixation (with cast, bandage, or splint), pinning, wiring, peripheral neurectomy, or a combination of these procedures. No reports are available on evidence of contracture formation over time in central paralysis (a MEDLINE search for articles published from 1966 to April 2004).

Many investigators (Evans et al. 1960; Furlow and Peacock 1965; Finsterbush and Friedman 1973; Woo et al. 1975; Langenskiöld et al. 1979; Wilson and Dahners 1988; Akai et al. 1993; Schollmeier et al. 1994; Reynolds et al. 1996; Trudel et al. 1999; Hildebrand et al. 2004) have measured ROM to assess how joint stiffness increases and angular displacement progressively decreases after immobilization. Evidence exists that contractures produced by immobilizing joints with internal fixation progress more rapidly at the beginning of the immobilization period (Trudel et al. 1999). In contrast, the actual rate of contracture progression following CNS injury is unclear. Data on the limitation in ROM over time are required to better understand this process and improve clinical management.

In the present study, we used, as representative models of animals with CNS injuries, rats with spinal cord injuries (SCI). Our objective was to determine the rate of contracture progression and the direction of loss in ROM following SCI.

\section{Materials ANd Methods}

This study was carried out after permission from the Committee of Research Facilities for Laboratory Animal Science, Hiroshima University School of Medicine.

\section{Animals}

Forty-eight female Wistar rats (CLEA Japan Incorporated, Tokyo), 8 weeks old, weighing 155 to $198 \mathrm{~g}$, were included in this study. Eight animals (4 experimental and 4 control) were studied at each of 5 time points: 2, 4, 8, 12, 16, and 24 weeks after surgical intervention. Rats were maintained at $23 \pm 1^{\circ} \mathrm{C}$, with a constant humidity of $55 \pm 5 \%$, a 12 hours light/dark cycle, and free access to food and water.

\section{Surgical procedures}

The weight was recorded for the 24 experimental animals and they were then anesthetized by intraperitoneal administration of $40 \mathrm{mg} / \mathrm{kg}$ sodium pentobarbital. A midline incision was made through the skin in the distal thoracic area. The lateral paraspinous muscles were dissected along either side of the spinous processes, exposing the dorsal surface of the T7 to T11 vertebrae. The spinal cord was exposed by a laminectomy of the T8 vertebrae. Using a no. 11 scalpel blade, the spinal cord was completely transected at the level of T8. Finally, the muscles and skin were closed separately with silk suture. The weight was also recorded for the 24 rats in the control group and then they were anesthetized with the same quantity of intraperitoneal pentobarbital as the experimental group. A sham-operation was carried out in which a laminectomy of the T8 vertebrae was performed but with no spinal cord transection.

\section{Postoperative care}

The condition of all animals was observed every day throughout the experimental period. Further, the bladders of the experimental animals were compressed manually twice daily. To prevent urine scald, the skin was wiped twice daily 
with diluted $0.1 \%$ chlorhexidine diacetate (Sigma Chemical Co., St. Louis, MO, USA) (Khan et al. 1999). During surgery and throughout the experimental period, the knee joint was left untouched.

\section{Behavioral assessment}

Before surgery and daily thereafter, behavioral observations were made. The following behavioral tests were performed before surgery and at the end of each experimental period: the BassoBeattie-Bresnahan (BBB) scale assessing the animal's gross hindlimb locomotor ability, ranging from 0 (flaccid paralysis) to 21 (normal walking) (Basso et al. 1995); Withdrawal reflexes assessing contact righting reflex and spinal cord-mediated hindlimb withdrawal reactions to extension, pain, and pressure, ranging from 0 (no reflexes) to 3 (hyperreflexia) (Gale et al. 1985). The behavioral tests we used have been commonly employed by other investigators to assess functional recovery after SCI (Gale et al. 1985; Basso et al. 1995; van de Meent et al. 1996; Khan et al. 1999). Only the highest point of motor performance was recorded during the 5 minutes of the tests. Behavioral observation was performed and behavioral tests were graded at the same time each day by the same examiner. During behavioral assessments, the animals were handled very carefully so to avoid hindlimb stimulation.

\section{Range of motion measurements}

After all behavioral assessments were completed, the weights of all the animals were recorded. The animals were then anesthetized with 40 $\mathrm{mg} / \mathrm{kg}$ sodium pentobarbital (i.p.). The lateral femoral condyle was the pivot point while the femur was fixed, and $14.60 \mathrm{~N} \cdot \mathrm{mm}$ flexion and extension moments were used on the tibia. The degree of contractures was assessed by measuring with a goniometer the femorotibial angle of both hindlimbs. In the present study, ROM measurements, as established by Wilson and Dahners (1988), were modified and used in measuring the degree of flexion and extension contractures. Briefly, the modification applied was to measure the full knee joint ROM of anaesthetized animals. The right and the left knee joint served as different samples. Two examiners conducted the ROM measurements and repeated them ten times for each leg for both flexion and extension. Values were the mean of the 20 measurements, the combined measurements taken by both examiners. The total ROM was the sum, in absolute values, of the angular displacement in flexion and in extension.

\section{Statistical analyses}

Analyses were performed with SPSS 11.5J for Windows (SPSS Japan Incorporated, Tokyo). The amount of weight gained and results of ROM measurements are presented as mean \pm S.D. To evaluate the amount of weight gained as an indicator of the animal's health, and to determine the degree of contracture and the progression of the knee joint contracture over time, comparisons between the groups and within each group were performed using two-way analysis of variance (ANOVA). When significant main effects or interactions were found, analyses with simple main effects or a least significant difference post-hoc test followed ANOVA to locate the levels of factor(s) with significant differences in the means of the variables. Behavioral scores are presented as medians, with interquartile ranges. Differences between the experimental and control group were compared at each time point by Mann-Whitney's U-test. We assessed behavioral changes over time within each group by using the Kruskal-Wallis test with post-hoc Mann-Whitney's U-tests. Posthoc correction for multiple comparisons was applied using the control for False Discovery Rate (Benjamini and Hochberg 1995). An alpha of less than 0.05 was chosen as the significance level for all statistical analyses.

\section{Results}

The weight of the experimental animals significantly decreased at all time points $\left(F_{(1,36)}\right.$ $=72.26, p<0.001)$. No significant interaction was detected between the group and the amount of 
weight gained over time $\left(F_{(5,36)}=0.88, p=0.51\right)$, and therefore animals in the experimental and control groups grew similarly over time.

\section{Behavioral outcome}

Rats with SCI crept, and dragged the hindlimbs throughout the experimental period. The response to stimuli disappeared, and animals demonstrated complete flaccid paraplegia during the first few days after the injury. Slight movement of hip and/or knee joints was apparent by 1 to 2 weeks postinjury. Clonic, high-frequency flexion-extension movement in the knee and ankle joint simultaneously occurring in combination with hyperflexion of the hip joint was often observed after 2 weeks. Hindlimb extension with rapid myospasm and toe abduction was observed at the beginning of advancement after the slight movement of hip and/or knee joints. The results of BBB scores indicated that no significant changes in recovery were observed after 2 weeks for the animals with SCI (Fig. 1). The withdrawal reflexes scores indicated that the experimental animals with SCI had a significant hyperreflexive response compared with all the animals presurgery and with the control group postsurgery $(p<0.05$; the results were not shown). All animals before surgery demonstrated normal behavior, and the control animals had no observable deficits at any time during the experimental period after laminectomy.

\section{Progressive process of contractures}

ANOVA confirmed a significant group-bytime interaction for total $\operatorname{ROM}\left(F_{(5,84)}=77.31\right.$, $p<0.001)$. Knee joint ROM of the experimental animals was significantly more restricted than that of the control animals at all time points $(p<0.001)$. The contractures progressed significantly for the first 12 weeks after SCI $(\mathrm{MSe}=8.12, p<0.01)$. No further loss in ROM was detected between weeks 12 and 24 (MSe=8.12, $p>0.05$ ) (Fig. 2). The average rate of contracture progression was $8.58^{\circ}$ weekly for the first 2 weeks. ROM restriction decreased gradually from $5.27^{\circ}$ to $1.05^{\circ}$ weekly between weeks 2 and 8 . However, the progressive restriction in total ROM increased again by $3.36^{\circ}$ weekly between weeks 8 and 12. After that, the contracture progression after 12 weeks, when no restriction in total ROM could be detected, was almost $0^{\circ}$.

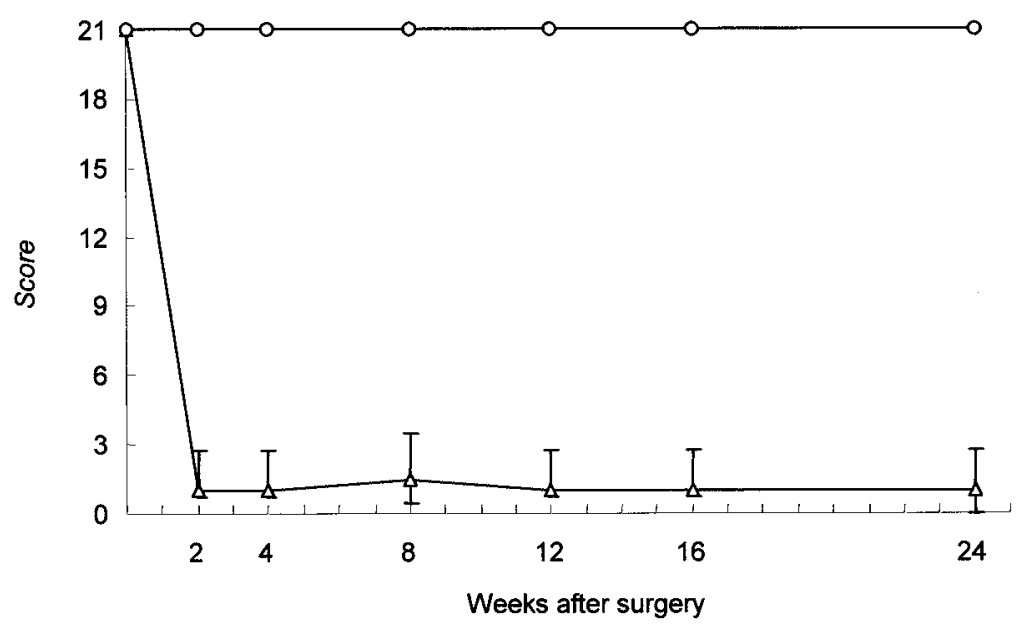

Fig. 1. Locomotor changes over time shown on the BBB scale. Performance of the animals with SCI was significantly lower than that of control animals $(p<0.05)$. Rats with SCI crept and demonstrated slight movement of the hindlimbs throughout the experimental period. No significant changes in neurologic function could be seen between week 2 and week 24, and performance recovery reached a plateau by week 2 . Error bars represent the 25 th to 75 th percentile. $\bigcirc-\bigcirc$, control; $\triangle-\triangle$, experimental. 


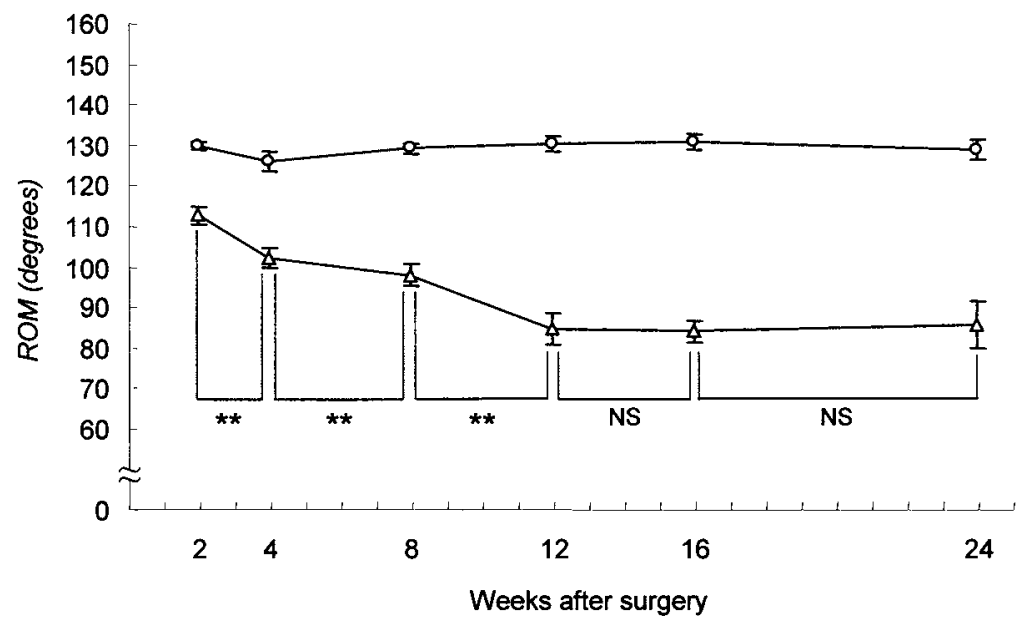

Fig. 2. Contracture progression following SCI over time. The ROM in the knees of the experimental rats was restricted significantly at all time points. The contractures progressed during the first 12 weeks and plateaued thereafter. Error bars represent \pm 1 s.D. NS, not significant. ${ }^{* *} p<0.01$. $\bigcirc-\bigcirc$, control; $\triangle-\triangle$, experimental.

\section{Restricted direction of contractures}

There was a significant interaction for flexion $\operatorname{ROM}\left(F_{(5,84)}=3.22, p=0.01\right)$ and extension $\operatorname{ROM}\left(F_{(5,84)}=150.67, p<0.001\right)$. In the comparison of extension ROM between the groups, the ROM of the experimental animals was restricted significantly at all time points $(p<0.001)$. In con- trast, flexion ROM increased significantly at 2, 4, 8 , and 24 weeks $(p<0.01)$, although no statistically significant differences were found at 12 and 16 weeks $(p>0.05)$ (Fig. 3). Consequently, the contractures were produced almost exclusively by a loss in the extension ROM.

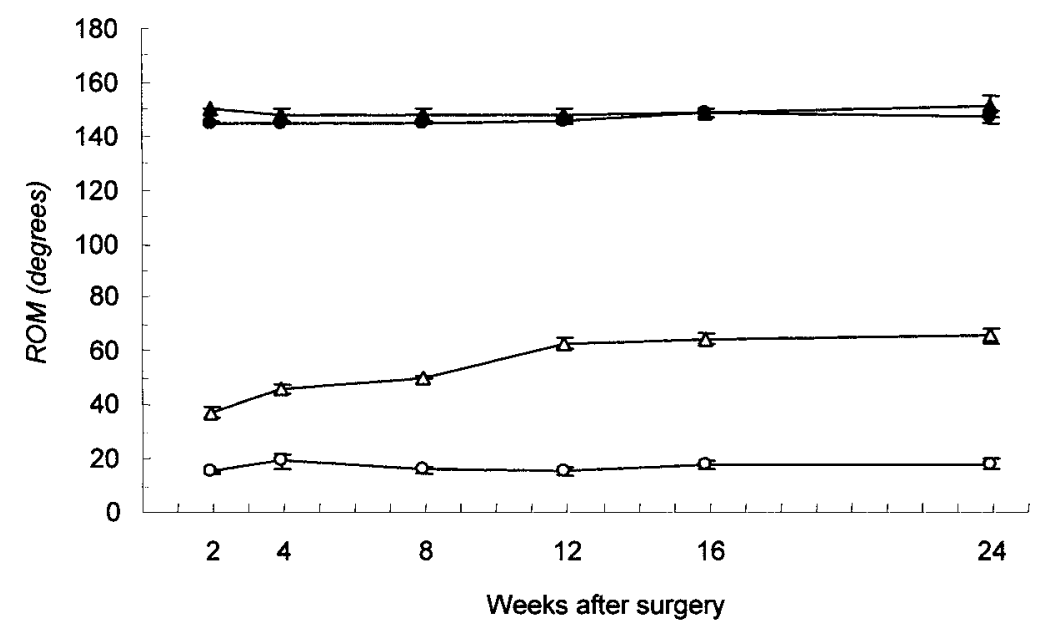

Fig. 3. Direction of the restriction in angular displacement. At all time points, extension ROM in the knee joints of the experimental animals was restricted greatly but flexion ROM differed only slightly from that of the control animals. Error bars represent \pm 1 s.D. -0 , control flexion; experimental flexion; $\bigcirc-\bigcirc$, control extension; $\triangle-\triangle$, experimental extension. 


\section{Discussion}

The rate of contracture progression was more rapid in the early stage after SCI and stabilized in the later stage of the injury. Contractures following SCI occurred in flexion at the knees and resulted from a loss of extension.

\section{Internal validity}

The animals with SCI lost weight as a result of muscle atrophy (Okumura 1997), but grew as well as the control animals. These results suggest general good health except for the hindlimb functional deficits associated with SCI and further suggest no difference in conditions (e.g., pain, decrease in level of activity, or nutrition) during the experimental period.

\section{Behavioral analysis}

Our experimental animals with SCI demonstrated a complete flaccid paralysis during the first few days postsurgery and thereafter showed kick movements, clonic and high-frequency flexionextension movements, and hyperreflexive response which were defined as spasticity (van de Meent et al. 1996). Therefore, the experimental animals demonstrated a condition such as that reported in humans following CNS injuries, who exhibit complete flaccid paralysis soon after the injury followed by the appearance of spastic paralysis (Botte et al. 1988), as reported in other studies (Gale et al. 1985; Basso et al. 1995; van de Meent et al. 1996; Khan et al. 1999).

\section{Progression of contractures over time}

Flexion contractures are the most common knee abnormalities related to CNS disorders (Banks 1972; Morrell et al. 2002; Kagaya et al. 2003). Knee flexion contractures occur in animals with SCI as well as in humans. In the behavioral observations, the hindlimbs were dragged behind the animals. It is interesting, nevertheless, that flexion contractures developed; this mechanism was not elucidated by this study. Although knee flexion contractures in humans are associat- ed with hip and ankle flexion contractures (Banks 1972; Chambers 2001), these dynamic conditions may not be applicable because rats were quadrupeds and the spinal cord-injured rats dragged their hindlimbs. The anatomic constitution of the rat knee is very similar to that of the human knee (Evans et al. 1960). Therefore, a rat with SCI is presumably a more appropriate model than other animals with immobilized joints in the elucidation of contractures with different developmental mechanisms.

Trudel et al. (1999) immobilized rat knees with extraarticular internal fixation for 2, 4, 8, 16 and 32 weeks, and provided quantitative data on ROM. They identified two phases, an acute phase that progressed for the first 16 weeks and a chronic phase that finally reached a steady state thereafter. The present study is the first to provide information on the progression of contractures over time following SCI.

Contractures after immobilization mainly result from stress deprivation of joints (Akeson et al. 1987; Trudel et al. 1999). In addition, contractures following CNS injuries have another developmental factor associated with muscle tone (Sharrard 1967; Banks 1972; Botte et al. 1988; Keenan et al. 1988; Farmer and James 2001). In an acute stage, during which flaccidity is evident, the joint immobility triggers contractures. Loss of elasticity and shortening may develop in tendons, fasciae, intermuscular septa and capsules, and in ligaments in which collagen is deposited; the changes occur too soon and too rapidly to be caused by disuse and adaptation (Sharrard 1967). In the subsequent stage in which spasticity develop, hypertonicity is mostly implicated in contracture development. Spasticity produces muscular imbalance when the spastic muscle continuously contracts, pulling the affected limb into a posturing or fixed position (Sharrard 1967; Farmer and James 2001). If the limb is allowed to remain fixed in the postured position, contractures will develop (Botte et al. 1988; Farmer and James 2001). Based on these studies, immobilization caused by spasticity may produce contractures. 
We observed that the animals with SCI showed some movement of knee joints. However, the contracture process following SCI was similar to that of rigid internal immobilization, and the period when the restriction in ROM reached a plateau was similar (Trudel et al. 1999). Therefore, the present results suggest that factors other than immobilization take part in contracture development. In view of the limitation of joint movement, these findings suggest that various therapeutic interventions (stretching, pharmacologic treatments, or surgery) have to be timed to match the optimal phase of contracture. This aspect may be crucial to improving clinical management.

In contractures occurring long after the onset of central paralysis, Enneking and Horowitz (1972) observed that a patient with traumatic paraplegia of three and one-half years' duration had knee ROM of $30^{\circ}$ (from $60^{\circ}$ to $90^{\circ}$ ), and Keenan et al. (1988) reported that the average degree of flexion contracture was $61.4^{\circ}$ in 46 human knees 3 years, on average, after onset of spasticity. Contractures following CNS injuries result from diverse causes, and some treatments are given according to the severity of the deformity. Although generalization of this study's findings may be limited by the species barrier, we conjecture that contractures gradually progress to the state presented if they occur in disorders of longer duration, because ROM of $86^{\circ}$ at 24 weeks was still present.

The contracture process animals exhibit after immobilization is very similar to that reported in humans (Trudel et al. 1999). Whether our results can be applied to humans with SCI or to other CNS disorders remains controversial, but warrants future examination. Also, it would be useful to further investigate how each evolves over time to reach ankylosis beyond 24 weeks.

\section{Conclusion}

This study provides evidence that knee flexion contractures progress more rapidly in the early stage after SCI. These findings should help guide timely treatment and provide a better understand- ing of contracture development.

\section{Acknowledgments}

We appreciate the advice and expertise of Dr. Seiichi Kawamata. Gratitude is expressed to Eriko Tsutsumi and Junya Ozawa for technical assistance, Naohiko Kanemura and Makoto Watanabe for help with the experiment, and Ryuji Kobayashi and Kunji Shirahama for critical advice. We also thank Dr. Isao Nara for critical review of the manuscript.

\section{References}

Akai, M., Shirasaki, Y. \& Tateishi, T. (1993) Viscoelastic properties of stiff joints: a new approach in analyzing joint contracture. Biomed. Mater. Eng., 3, 67-73.

Akeson, W.H., Amiel, D., Abel, M.F., Garfin, S.R. \& Woo, S.L. (1987) Effects of immobilization on joints. Clin. Orthop., 219, 28-37.

Banks, H.H. (1972) The knee and cerebral palsy. Orthop. Clin. North Am., 3, 113-129.

Basso, D.M., Beattie, M.S. \& Bresnahan, J.C. (1995) A sensitive and reliable locomotor rating scale for open field testing in rats. J. Neurotrauma, 12, 1-21.

Benjamini, Y. \& Hochberg, Y. (1995) Controlling the False Discovery Rate: a practical and powerful approach to multiple testing. J. R. Statist. Soc. B., 57, 289-300.

Botte, M.J., Nickel, V.L. \& Akeson, W.H. (1988) Spasticity and contracture. Physiologic aspects of formation. Clin. Orthop., 233, 7-18.

Chambers, H.G. (2001) Treatment of functional limitations at the knee in ambulatory children with cerebral palsy. Eur. J. Neurol., 8, 59-74.

Enneking, W.F. \& Horowitz, M. (1972) The intra-articular effects of immobilization on the human knee. J. Bone Joint Surg. Am., 54, 973-985.

Evans, E.B., Eggers, G.W.N., Butler, J.K. \& Blumel, J. (1960) Experimental immobilization and remobilization of rat knee joints. J. Bone Joint Surg. Am., 42, 737-758.

Farmer, S.E. \& James, M. (2001) Contractures in orthopaedic and neurological conditions: a review of causes and treatment. Disabil. Rehabil., 23, 549-558.

Finsterbush, A. \& Friedman, B. (1973) Early changes in immobilized rabbits knee joints: a light and electron microscopic study. Clin. Orthop., 92 , 305-319.

Furlow, L.T., Jr. \& Peacock, E.E., Jr. (1965) Effect of beta-amino propionitrile on the prevention and 
treatment of joint stiffness in rats. Surg. Forum., 16, 457-458.

Gale, K., Kerasidis, H. \& Wrathall, J.R. (1985) Spinal cord contusion in the rat: behavioral analysis of functional neurologic impairment. Exp. Neurol., 88, 123-134.

Hildebrand, K.A., Sutherland, C. \& Zhang, M. (2004) Rabbit knee model of post-traumatic joint contractures: the long-term natural history of motion loss and myofibroblasts. J. Orthop. Res., 22, 313-320.

Kagaya, H., Ito, S., Iwami, T., Obinata, G. \& Shimada, Y. (2003) A computer simulation of human walking in persons with joint contractures. Tohoku. J. Exp. Med., 200, 31-37.

Keenan, M.A., Ure, K., Smith, C.W. \& Jordan, C. (1988) Hamstring release for knee flexion contracture in spastic adults. Clin. Orthop., 236, 221-226.

Khan, T., Havey, R.M., Sayers, S.T., Patwardhan, A. \& King, W.W. (1999) Animal models of spinal cord contusion injuries. Lab. Anim. Sci., 49, 161-172.

Langenskiöld, A., Michelsson, J.E. \& Videman, T. (1979) Osteoarthritis of the knee in the rabbit produced by immobilization. Attempts to achieve a reproducible model for studies on pathogenesis and therapy. Acta Orthop. Scand., 50, 1-14.

Morrell, D.S., Pearson, J.M. \& Sauser, D.D. (2002) Progressive bone and joint abnormalities of the spine and lower extremities in cerebral palsy. Radiographics, 22, 257-268.

Okumura, H. (1997) Experiment osteoporosis in the rat: immobilization osteoporosis. In: Handbook of Bone Morphometry, 2nd ed., edited by $\mathrm{H}$. Takahashi, Nishimura Co., Niigata, pp. 134-141. (in Japanese with English abstract)

Reynolds, C.A., Cummings, G.S., Andrew, P.D. \& Tillman, L.J. (1996) The effect of nontraumatic immobilization on ankle dorsiflexion stiffness in rats. J. Orthop. Sports Phys. Ther., 23, 27-33.

Schollmeier, G., Uhthoff, H.K., Sarkar, K. \& Fukuhara, K. (1994) Effects of immobilization on the capsule of the canine glenohumeral joint. A structural functional study. Clin. Orthop., 304, 37-42.

Sharrard, W.J. (1967) Paralytic deformity in the lower limb. J. Bone Joint Surg. Br., 49, 731-747.

Trudel, G., Uhthoff, H.K. \& Brown, M. (1999) Extent and direction of joint motion limitation after prolonged immobility: an experimental study in the rat. Arch. Phys. Med. Rehabil., 80, 1542-1547.

van de Meent, H., Hamers, F.P., Lankhorst, A.J., Buise, M.P., Joosten, E.A. \& Gispen, W.H. (1996) New assessment techniques for evaluation of posttraumatic spinal cord function in the rat. $J$. Neurotrauma, 13, 741-754.

Wilson, C.J. \& Dahners, L.E. (1988) An examination of the mechanism of ligament contracture. Clin. Orthop., 227, 286-291.

Woo, S.L., Matthews, J.V., Akeson, W.H., Amiel, D. \& Convery, F.R. (1975) Connective tissue response to immobility. Correlative study of biomechanical and biochemical measurements of normal and immobilized rabbit knees. Arthritis Rheum., 18, 257-264. 